\title{
Protein delivery options: how well have we succeeded?
}

\author{
"Protein and peptide therapeutics are revolutionizing treatment options for various \\ diseases. However, without appropriate sustained-delivery options, we are not \\ taking full advantage of their potency.
}

\begin{abstract}
Keywords: inhalation $\bullet$ innovations $\bullet$ in situ polymer precipitation $\bullet$ microneedle - painless routes $\bullet$ PLGA microsphere $\bullet$ protein $\bullet$ recombinant antibodies

- semi-automated injectors $\bullet$ sustained delivery
\end{abstract}

Of the ten top-selling drugs in 2013, the first three (Humira ${ }^{\circledR}$, Enbrel $^{\circledR}$, Remicade ${ }^{\circledR}$ ) were all monoclonal antibodies for treating autoimmune diseases; together these three accounted for approximately $\$ 28$ billion in sales. Another antibody, Avastin ${ }^{\circledR}$, indicated for bowel cancer, accounted for $\$ 6.7$ billion among these top-selling drugs, while Herceptin $^{\circledR}$, used to treat breast cancer, had sales of $\$ 6.6$ billion. These numbers bear eloquent testimony to the increasing demand for protein therapeutics. All of these proteins are made by recombinant synthesis which has truly revolutionized the ready availability of human analogs.

Such antibodies are administered via subcutaneous injection or via intravenous infusion; the frequency of administration varies. For example, Enbrel is injected subcutaneously once a week for rheumatoid arthritis whereas Remicade dosing is $3 \mathrm{mg} / \mathrm{kg}$ given via intravenous induction at 0,2 and 6 weeks. While the number of protein therapeutics has grown substantially over the last decade [1], delivery systems continue to be limited. We examine the reasons for this and discuss what we can expect in the future.

Innovations in delivery system are driven by the following factors:

- Greaterease-of-use(patient-friendliness).

- Reduced frequency of administration (sustained delivery).

- Less painful routes of administration.
With proteins, there are specific issues that make innovation tricky. For example, denaturation is a problem that limits longterm storage under ambient conditions, in an injectable form. Thus, several protein therapeutics are packaged as lyophilized powders that must be reconstituted prior to use, which detracts from user-friendliness. Insulin in solution form is kept in sealed cartridges, and usually refrigerated prior to injection. For the same reason, protein therapeutics must also avoid exposure to organic solvents and high temperatures during preparation and processing.

Instability in low-pH is another major drawback for proteins, one that precludes administration in oral tablet or capsule form. It is generally agreed that most patients prefer oral administration over all other forms, particularly for chronic conditions. Yet, not a single major protein therapeutic has been approved for oral administration to date.

The permeability of mucosal surfaces to proteins is usually much lower than for low molar mass drugs, due mainly to the larger size. This is also a limiting factor for alternative routes of administration, such as oral or topical dosage forms (transdermal and transcorneal, in particular) which are very useful for low molar mass drugs.

Finally, immunogenicity is more of an issue with protein therapeutics than with small molecules. Thankfully, this problem has been anticipated early on in the development process, so that for the

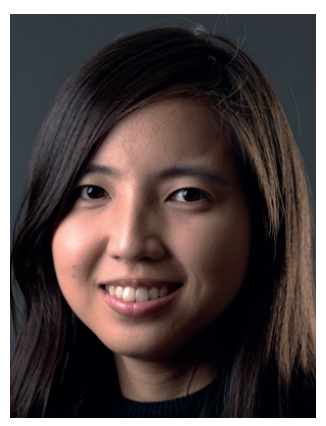

$\mathrm{Ng}$ Xu Wen

School of Materials Science \& Engineering, Nanyang Technological University, Block N4.1-02-06, Nanyang Avenue, Singapore 639798

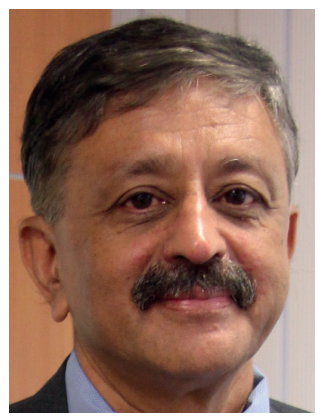

Subbu Venkatraman Author for correspondence: School of Materials Science \& Engineering, Nanyang Technological University, Block N4.1-02-06, Nanyang Avenue, Singapore 639798

Tel.: +65 67904259

assubbu@ntu.edu.sg 
approved proteins, large-scale exposure has not led to severe allergic reactions.

\section{Greater ease of use}

The one major direction of research into this aspect involves semiautomated injection systems. In particular, autoinjectors have been developed for a few of the major protein therapeutics. In the case of Humira, or adalimumab, the conventional treatment had been to administer the doses once or twice a week via subcutaneous injection. For this chronic treatment, the Humira Pen was developed as a disposable, prefilled system, that resembles a pen with a disposable needle not visible to the patients. In a trial with selected patients (Trial of Usability in Clinical Setting of Humira Pen, also known as TOUCH), $88 \%$ preferred the Pen to a prefilled syringe, and $77 \%$ found it less painful [2].

This led to similar autoinjectors being developed, most notably for Insulin and for etanercept (Enbrel), two proteins relatively stable to storage in solution. For arthritis sufferers, in particular, these autoinjectors are a boon.

\section{Painless routes of administration}

By far, the greatest activity has been on the inhalation concept for delivering (in particular) insulin. For a while, it has been known that protein adsorption in the lungs is facilitated via transcytosis and/or paracellular transport. Pfizer introduced Exubera ${ }^{\circledR}$ after 11 years of development with Nektar. This is a dry powder inhaler that delivers insulin relatively painlessly and with fairly rapid onset of action (compared with subcutaneous delivery). Nevertheless, the product was not a commercial success, primarily due to poor patient acceptance, resulting substantially from the bulkiness of the inhaler device. Exubera was removed from the market barely a year after its introduction [3].

\section{"The mainstay of administration continues to be injections (intravenous, subcutaneous, intramuscular) while other modes (inhalation, transdermal, even oral) are being explored currently."}

However, the inhalation route has not been completely abandoned. Using personal funds, Alfred Mann founded Mannkind to develop the nextgeneration insulin inhaler, named Afrezza, which has recently won US FDA approval for Type 1 diabetes. The Afrezza technology also relies on powder inhalation, but the device is much more compact and easier to use, and breath activated, which allows for more accurate dosing.
The use of 'microneedles' in a skin patch is another less painful alternative compared with subcutaneous injection, and this approach has been explored by several researchers [4-6]. The most studied approach is delivering a bolus of inactivated virus as a vaccine by this route $[4,7]$, and mice studies have shown good efficacy, although human trials have not yet been reported. The main advantage of this approach appears to be the convenience of administration in the field, particularly in remote areas, rather than the painlessness itself. For this reason, perhaps, vaccination via a patch has not attracted funding from Big Pharma.

For nonvaccine delivery, a company called Zosano Pharma (ZP) appears to be heading the field. The company has reported Phase II trial for their technology (which the website refers to as a needle-free patch, but nevertheless uses microneedles) to deliver a teriparatide parathyroid hormone $(\mathrm{PTH})$ for severe arthritis (Zosano Pharma-(ZP)-PTH in partnership with Eli Lilly), and glucagon for severe hypoglycemia (ZPglucagon, no partner yet). The needles or microprojections are made of titanium metal and the needles are coated with the bioactive molecule. Storage stability is a big issue, but for the PTH, and for glucagon, shelf-lives of 36 months are reported [8,9].

Both the inhalation and microneedle approaches are not particularly suited to sustained delivery of proteins, which is the third goal of innovative protein delivery approaches. For this, the main options appear to be microparticles and in situ gelation systems, both administered via subcutaneous injections. A hugely successful product that uses biodegradable microspheres for delivering leuprolide acetate (LA; a synthetic nonapeptide analog of gonadotropin releasing hormone) is TAP pharmaceutical's Lupron Depot. This product is available in a monthly, 3-monthly and 6-monthly (intramuscular) injection form (for prostate cancer), indicating the long-term sustained effect of leuprolide acetate (LA). The release is governed initially by diffusion through the poly(lactide-coglycolide) (PLGA) microspheres, and subsequently via degradation of the polymer.

This success story has not been duplicated with other peptides or proteins. In fact, sustained delivery of LA has been replicated by another technology called in situ polymer precipitation developed at Atrix Labs in the 1980s into a product called Eligard ${ }^{\circledR}$, also for prostate cancer management (Atrix Labs was acquired by QLT, Inc., in 2004, and only the ELigard product is now marketed by QLT). The Atrigel technology is simple and straightforward to manufacture, as it consists of a solution of the carrier polymer and the LA that is injected subcutaneously. The solution under- 
goes solvent-nonsolvent exchange with water from tissue, resulting in 'in situ precipitation' with consequent control over drug (or protein) release. A major drawback of this system has been the substantial early (or burst) release [10] but later modifications have led to much better control over the burst [11]. Issues with biocompatibility of the organic solvent remain, and have prompted the development of another system based on sucrose acetate isobutyrate or SAIB which forms a viscous depot upon injection from an alcohol solvent medium. SAIB is a viscous liquid that is generally recognized as safe (GRAS) by the FDA, and used commonly as a food additive. The addition of a solvent such as ethanol decreases its viscosity dramatically enough for easy injectability. The drug or protein may be dissolved or suspended in a SAIB solution prior to injection. This technology is the basis for nonprotein product application (bupivacaine, POSIDUR ${ }^{\circledR}$ NDA filed 2014) but is touted for protein delivery as well [12]. The company (DURECT Corporation) claims that the SABER approach decreases burst delivery of proteins as compared with PLGA microsphere delivery, but it is hard to see why this is so, or can be so for a broad range of proteins.

\section{Conclusion}

Protein and peptide therapeutics are revolutionizing treatment options for various diseases. However, without appropriate sustained-delivery options, we are not taking full advantage of their potency. A few examples of such sustained-delivery options have been commercialized but more needs to be done to expand the scope of user-friendly and localized delivery options. One

\section{References}

1 Pavlou AK, Reichert JM. Recombinant protein therapeuticssuccess rates, market trends and values to 2010. Nat. Biotech 22(12), 1513-1519 (2004).

2 Kivitz A, Cohen S, Dowd JE et al. Clinical assessment of pain, tolerability, and preference of an autoinjection pen versus a prefilled syringe for patient self-administration of the fully human, monoclonal antibody adalimumab: the TOUCH trial. Clin. Ther. 28(10), 1619-1629 (2006).

3 Mack GS. Pfizer dumps Exubera. Nat. Biotech. 25(12), 1331-1332 (2007).

4 Sullivan SP, Koutsonanos DG, Del Pilar Martin M et al. Dissolving polymer microneedle patches for influenza vaccination. Nat. Med. 16(8), 915-920 (2010).

5 Cormier M, Johnson B, Ameri M et al. Transdermal delivery of desmopressin using a coated microneedle array patch system. J. Control Release 97(3), 503-511 (2004).

6 Banga AK. Microporation applications for enhancing drug delivery. Expert Opin. Drug Deliv. 6(4), 343-354 (2009). important issue with all current delivery options is the 'initial burst' release of the therapeutic, resulting in unwanted side effects, as well as wastage of the therapeutic entity. This is an important issue that needs to be addressed especially for protein drugs with a narrow therapeutic window.

\section{Future perspective}

Delivery options for proteins have not kept pace with the burgeoning field of novel protein therapeutics. The mainstay of administration continues to be injections (intravenous, subcutaneous, intramuscular) while other modes (inhalation, transdermal, even oral) are being explored currently. Nevertheless, the latter are mostly in the preclinical stage as of this writing. Breakthroughs are to be hoped for in applying nanotechnology to delivering proteins, either via active targeting or in less invasive modes of administration, particularly in oral tablets/capsules. If oral delivery becomes a reality, patient acceptance of protein therapeutics will be significantly increased leading to overall better disease management, as well as reduction in healthcare costs.

\section{Financial \& competing interests disclosure}

The authors have no relevant affiliations or financial involvement with any organization or entity with a financial interest in or financial conflict with the subject matter or materials discussed in the manuscript. This includes employment, consultancies, honoraria, stock ownership or options, expert testimony, grants or patents received or pending, or royalties.

No writing assistance was utilized in the production of this manuscript.

7 Vaxxas Nanopatch.

www.vaxxas.com

8 Zosano Pharma-ZP-PTH.

www.zosanopharma.com

9 Daddona PE, Matriano JA, Mandema J, Maa YF. Parathyroid hormone (1-34)-coated microneedle patch system: clinical pharmacokinetics and pharmacodynamics for treatment of osteoporosis. Pharm. Res. 28(1), 159-165 (2011).

10 Wang L, Venkatraman S, Kleiner L. Drug release from injectable depots: two different in vitro mechanisms. J. Control Release 99(2), 207-216 (2004).

11 Lim SG, Ameenuddin S, Burnett JC Jr, Venkatraman SS, Chen HH. Sustained delivery of a novel natriuretic peptide for three weeks with in situ polymer precipitation delivery system. J. Card. Fail. 18(8), S63 (2012).

12 Durect. www.durect.com 